\title{
Low Loss, Wide Bandwidth, Low Bend Sensitivity HC-PBGF for Mid-IR Applications
}

\author{
N. V. Wheeler, A. M. Heidt, N. K . Baddela, J. R. Hayes, S. R. Sandoghchi, E. Numkam Fokoua, F. Poletti, \\ M. N. Petrovich, D. J. Richardson \\ Optoelectronics Research Centre, University of Southampton, Southampton SO17 1BJ, United Kingdom \\ n.v.wheeler@soton.ac.uk
}

\begin{abstract}
Hollow core-photonic bandgap fiber, operating in the spectral region between 3 and 4 $\mu \mathrm{m}$, is reported. Order of magnitude loss reduction is demonstrated $(0.13 \mathrm{~dB} / \mathrm{m})$ compared to previously reported fibers, combined with very low bend sensitivity.

OCIS codes: (060.5295) Photonic crystal fibers; (060.2280) Fiber design and characterization
\end{abstract}

\section{Introduction}

Hollow core fibers have several applications in the mid-IR including gas sensing [1], gas lasers [2] and high power laser delivery for medical applications [3]. While soft glass based structures have been favored for transmission in this wavelength region, due to their lower material attenuation compared to more conventional silica beyond $2.5 \mu \mathrm{m}$ [4], several low loss hollow core silica-based fibers have recently been reported which deliver low loss guidance up to $4 \mu \mathrm{m}$ (e.g. [3,5,6]). In particular, Yu et al. [5] reported a loss of $34 \mathrm{~dB} / \mathrm{km}$ at $3.050 \mu \mathrm{m}$ in combination with a broadband low loss guidance region extending from 2.85 to $3.85 \mu \mathrm{m}$. This loss figure is remarkably low considering the attenuation of bulk dry synthetic silica (e.g. Suprasil F300 from Heraeus) ranges from $60 \mathrm{~dB} / \mathrm{m}$ up to over 600 $\mathrm{dB} / \mathrm{m}$ in this spectral region [4]. These recently reported fibers confine light in the fiber core through anti-resonance with the core surround and low loss is in part achieved through a large core diameter (eg. $94 \mu \mathrm{m}$ [5]) which minimizes overlap between the core modes and the silica regions of the fiber. This large diameter, while suited for some applications, can restrict the use of these fibers due to the challenge of low loss integration with existing fiber technologies. Furthermore, due to the guidance mechanism, these fibers are extremely bend sensitive, restricting device footprint and/or usable operating lengths.

Hollow core-photonic bandgap fibers (HC-PBGFs) provide an alternative mechanism for light confinement in an air-core fiber. Until now, the lowest reported loss in this spectral region was $\sim 1 \mathrm{~dB} / \mathrm{m}[3]$ in HC-PBGFs operating at wavelengths up to $3.4 \mu \mathrm{m}$. Here we report a 19c HC-PBGF achieving an order of magnitude reduction in loss $(0.13$ $\mathrm{dB} / \mathrm{m}$ at $3.33 \mu \mathrm{m}$ ) in combination with a wide operating bandwidth, extending from 3.1 to $3.7 \mu \mathrm{m}$, and which also exhibits very low bend sensitivity.

\section{Results}

The HC-PBGF has a 19c core design and was fabricated using the conventional two-step stack and draw technique. Differential pressures in the core and cladding regions of the structure were carefully controlled during the fiber drawing process to maintain an optimum core surround and to minimize the overlap between the core guided modes and the silica core surround. A scanning electron microscope image of the fabricated fiber is shown in Fig. 1(a); the core diameter is $54 \mu \mathrm{m}$ and the average hole-to-hole distance is $\sim 10 \mu \mathrm{m}$.

(a)

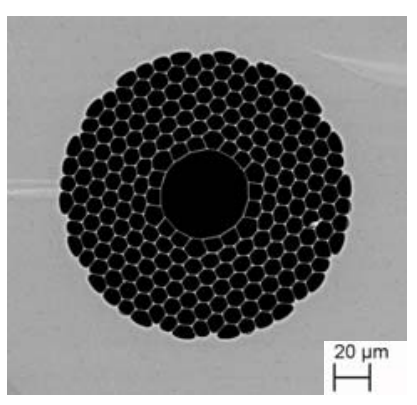

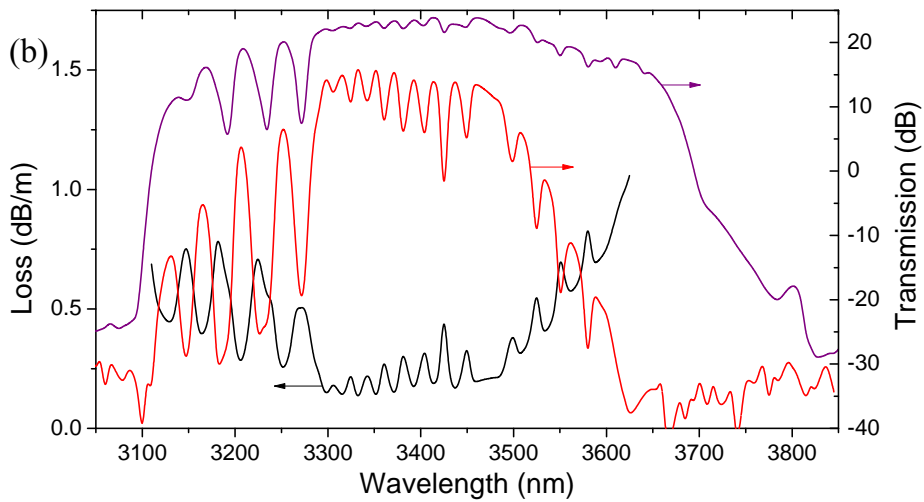

Fig. 1: (a) Scanning electron microscope image of HC-PBGF (b) Transmission through $58 \mathrm{~m}$ and $5 \mathrm{~m}$ of HC-PBGF shown by red line and purple line respectively. Cutback loss measurement (black line) indicates a minimum attenuation of $0.13 \mathrm{~dB} / \mathrm{m}$ at $3.33 \mu \mathrm{m}$. 
The fiber transmission (Fig. 1b) was recorded using a custom built supercontinuum source which generates a broad spectrum spanning from 0.75 to $4 \mu \mathrm{m}$ by pumping a ZBLAN fiber with a diode-seeded picosecond Thulium-doped fiber amplifier system at $2 \mu \mathrm{m}$ [7]. A monochromator was used for detection of the signal transmitted through the PBGF. A measurement over a $5 \mathrm{~m}$ fiber length shows that the bandgap extends from 3.1 to $3.8 \mu \mathrm{m}$ and a cutback loss measurement (58 to $5 \mathrm{~m}$ ) shows a minimum loss of $0.13(+/-0.05) \mathrm{dB} / \mathrm{m}$ at $3.33 \mu \mathrm{m}$ and $<1 \mathrm{~dB} / \mathrm{m}$ transmission loss over $>500 \mathrm{~nm}$ bandwidth despite the high bulk silica attenuation. This loss value indicates that the average overlap between the core guided modes and the silica surround is less than $0.3 \%$. At the short wavelength edge of the bandgap, there are several loss peaks which we believe are due to surface modes, while between 3.3 and $3.6 \mu \mathrm{m}$ many gas absorption lines are visible. These can be attributed to $\mathrm{HCl}$ absorption; this gas is present in the fiber as chlorine evolves over time from the silica and it can be removed by purging the fiber with nitrogen [5]. The measured minimum loss lies between two such absorption lines and it is likely that the loss value would be lower if the gas was eliminated from the fiber. Note that the measurement is limited by the $5 \mathrm{~nm}$ resolution of the monochromator. Furthermore, due to large mismatch between the launch ZBLAN fiber ( $9 \mu \mathrm{m}$ core diameter) and the PBGF, excitation of higher order modes in the latter is almost inevitable, leading to a loss overestimate. A wide guidance window was also observed in the near-IR spanning from 700 to $1400 \mathrm{~nm}$ which has a minimum transmission loss of $2.6 \mathrm{~dB} / \mathrm{m}$ at $1 \mu \mathrm{m}$. This opens up the possibility of simultaneous guidance of both near and midIR light in this fiber. The presence of resonant loss features within this band is indicative of anti-resonant (AR) guidance.

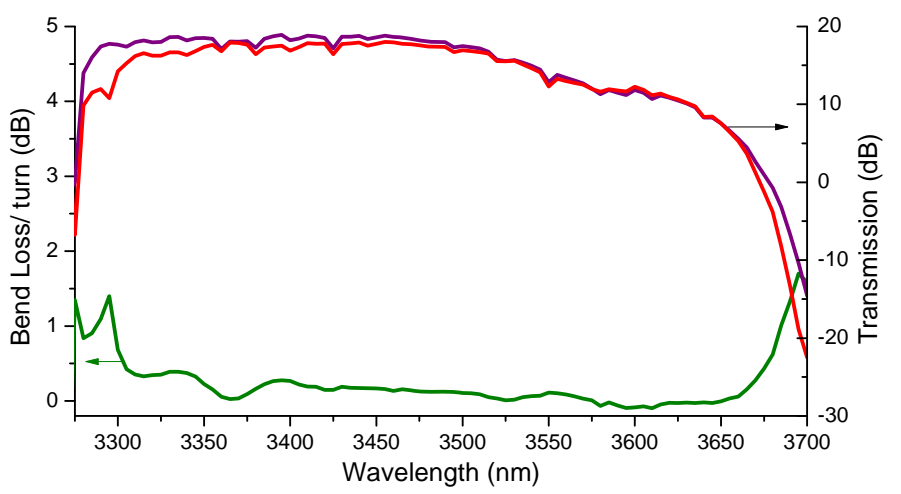

Figure 2: The transmission of $5 \mathrm{~m}$ of the HC-PBGF was recorded with 5 coils of $5 \mathrm{~cm}$ diameter in the fibre (red line) and with the fibre loosely coiled (purple line). The bend loss per turn (green line) is $<0.25 \mathrm{~dB}$ over a $300 \mathrm{~nm}$ bandwidth.

The bend loss of the HC-PBGF was measured by comparing the transmission through a $5 \mathrm{~m}$ length with 5 coils of 5 $\mathrm{cm}$ diameter and with the fiber only loosely coiled. These spectra, along with the resultant bend loss per turn are shown in Fig. 2. This measurement shows the bend loss is $<0.5 \mathrm{~dB} /$ turn over the entire surface mode free region of the bandgap and $<0.25 \mathrm{~dB} /$ turn over a $300 \mathrm{~nm}$ bandwidth. Note that this loss is significantly lower than that reported recently for AR fibers operating in the same wavelength region ( $\sim 6 \mathrm{~dB}$ for a $\sim 20 \mathrm{~cm}$ diameter coil [5]) and indicates that this fiber is well-suited for the fabrication of compact gas cells for sensing and spectroscopy.

\section{Conclusions}

We have fabricated and characterized a HC-PBGF which provides low loss guidance between 3.1 and $3.7 \mu \mathrm{m}$ with a record minimum attenuation of $0.13 \mathrm{~dB} / \mathrm{m}$ at $3.33 \mu \mathrm{m}$ for a HC-PBGF operating in the mid-IR. This low loss operation is combined with an extremely low bend loss for this spectral region of $<0.25 \mathrm{~dB}$ per $5 \mathrm{~cm}$ diameter turn over a $300 \mathrm{~nm}$ bandwidth. This work was supported by the UK EPSRC through grants EP/I01196X/1 (HYPERHIGHWAY) and EP/H02607X/1.

\section{References}

[1] L. Kornaszewski, et al., "Mid-infrared methane detection in a photonic bandgap fiber using a broadband optical parametric oscillator," Optics Express 15(18) pp. 11219-11224 (2007).

[2] A. M. Jones, et al., " $\mathrm{C}_{2} \mathrm{H}_{2}$ Gas Laser Inside Hollow-Core Photonic Crystal Fiber Based on Population Inversion," in CLEO, CTuU1 (2010).

[3] Urich, et al., "Delivery of high energy Er:YAG pulsed laser light at $2.94 \mu \mathrm{m}$ through a silica hollow core photonic crystal fiber," Optics Express 20(6), pp. 6677-6684 (2012).

[4] O. Humbach, et al., "Analysis of OH absorption bands in synthetic silica," J. Non-Cryst. Solids 203, 19-26 (1996).

[5] Yu, F., et al., "Low loss silica hollow core fibers for 3-4 $\mu \mathrm{m}$ spectral region," Optics Express, 20(10). pp. 11153-11158 (2012).

[6] A. N. Kolyadin, et al., "Light transmission in negative curvature hollow core fiber in extremely high material loss region," Optics Express,

21(8) pp. 9514-9519 (2013).

[7] A. M. Heidt, et al.,"100 kW peak power picosecond Thulium-doped fiber amplifier system seeded by a gain-switched diode laser at $2 \mu \mathrm{m}$,"

Opt. Lett. 38(10), pp. 1615-1617 (2013). 